Technological University Dublin

DÜBLIN

ARROW@TU Dublin

2010-01-01

\title{
Electronic Speckle Pattern Interferometer using Holographic Optical Elements for Vibration Measurements
}

\author{
Viswanath Bavigadda \\ Technological University Dublin \\ Raghavendra Jallapuram \\ Technological University of Dublin, mrutic@gmail.com \\ Emilia Mihaylova \\ Technological University of Dublin, emilia.mihaylova@tudublin.ie
}

See next page for additional authors

Follow this and additional works at: https://arrow.tudublin.ie/cieoart

\section{Recommended Citation}

Bavigadda, V. et. al. (2010) Electronic speckle pattern interferometer using holographic optical elements for vibration measurements. Optics Letters, Vol. 35, No. 19, October 1, pp.3273-3275. doi:10.1364/ OL.35.003273

This Article is brought to you for free and open access by the Centre for Industrial and Engineering Optics at ARROW@TU Dublin. It has been accepted for inclusion in Articles by an authorized administrator of ARROW@TU

Dublin. For more information, please contact

arrow.admin@tudublin.ie, aisling.coyne@tudublin.ie, gerard.connolly@tudublin.ie.

Funder: Enterprise Ireland

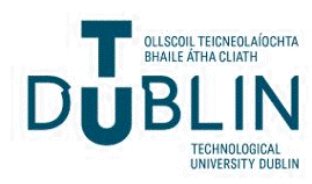


Authors

Viswanath Bavigadda, Raghavendra Jallapuram, Emilia Mihaylova, and Vincent Toal

This article is available at ARROW@TU Dublin: https://arrow.tudublin.ie/cieoart/69 


\title{
Electronic speckle pattern interferometer using holographic optical elements for vibration measurements
}

\author{
Viswanath Bavigadda*, Raghavendra Jallapuram, Emilia Mihaylova and Vincent Toal \\ Centre for Industrial and Engineering Optics, Dublin Institute of Technology, Kevin Street, \\ Dublin 08, Ireland \\ *Corresponding author: viswanath.bavigadda@student.dit.ie
}

\begin{abstract}
A simple and compact electronic speckle pattern interferometry (ESPI) built with holographic optical elements (HOEs) used for the study of out-of-plane vibration is reported. Carefully fabricated reflection and transmission HOEs provide reference and object beams in the interferometer. All the alignment difficulties in conventional ESPI systems are minimized using HOEs. The time average ESPI subtraction method is used to generate correlation fringes. The background speckle noise is removed by introducing a phase shift between sequential images. The amplitude and phase maps are obtained using path difference modulation in an unbalanced ESPI.
\end{abstract}

Electronic speckle pattern interferometry is a popular non-destructive testing tool for quantitative measurement of dynamic displacements of an object ${ }^{1,2}$. Conventional ESPI systems consist of expensive bulk optical elements whose alignment is a critical and time consuming task. Alternative ESPI systems were designed using diode lasers and single mode optical fibres ${ }^{3,4}$. ESPI systems can be further simplified by using holographic optical elements. in place of bulk optics. A HOE based ESPI system was reported by Petrov and Lau ${ }^{5}$. In-plane and out-of-plane 
sensitive ESPI systems using diffraction gratings ${ }^{6}$ and HOEs providing purely out of plane sensitivity in interferometric systems ${ }^{7,8,9}$ have also been reported. Time average ESPI with phase stepping is a widely used technique for vibration measurement ${ }^{1}$. Vibration may also be measured using the reference beam modulation technique ${ }^{10,11}$. In this letter, we report a HOE based ESPI system for out-of-plane vibration measurement. A reflection holographic optical element (RHOE) provides the reference beam in the interferometer ${ }^{12}$ and a transmission holographic optical element (THOE) is used to provide normal illumination of the test object ly, making the ESPI system sensitive only to out-of-plane displacement. The laser diode wavelength is modulated to provide path length modulation in the interferometer.

Speckle correlation fringes in ESPI are normally obtained by subtraction of two images corresponding to the displaced and undisplaced positions of the test object. When the test object is vibrating harmonically at an angular frequency $\omega$, amplitude $a_{0}$ and phase $\varphi_{0}$, the brightness at any point of the correlogram can be described as ${ }^{11}$

$$
B=4 \sqrt{I_{0} I_{r}}\left|J_{0}\left(\frac{4 \pi}{\lambda} a_{0}\right) \cos (\psi)\right|
$$

where the background intensity term $I_{0}+I_{r}$ due to object and reference beam intensities $I_{0}$ and $I_{r}$ has been removed as explained later.

To obtain contours of constant amplitude and phase, the reference beam is modulated at the same angular frequency as that of the test object $\omega$ but with an amplitude $a_{r}$ and phase $\varphi_{r}$, the brightness of the correlogram at any point is modified to become

$$
B^{\prime}=4 \sqrt{I_{0} I_{r}}\left|J_{0}\left(\frac{4 \pi}{\lambda}\left\{a_{0}^{2}+a_{r}^{2}-2 a_{0} a_{r} \cos \left(\varphi_{0}-\varphi_{r}\right)\right\}^{1 / 2}\right) \cos (\psi)\right|
$$


The brightness usually modulates over only a few gray levels, making it difficult hard to detect fringes visually so we simply square the brightness $B^{\prime}$ and display the result as

$$
B^{\prime \prime}=16 I_{0} I_{r}\left|J_{0}\left(\frac{4 \pi}{\lambda}\left\{a_{0}^{2}+a_{r}^{2}-2 a_{0} a_{r} \cos \left(\varphi_{0}-\varphi_{r}\right)\right\}^{1 / 2}\right) \cos (\psi)\right|^{2}
$$

As we vary the amplitude of the reference beam path modulation in the interferometer by varying the laser drive current, $i$, the regions where $a_{r}=a_{0}$ and $\varphi_{r}=\varphi_{0}$ are displayed with maximum brightness ${ }^{10,11}$.

A plot of wavelength versus drive current enables us to identify mode hop free regions of operation of a diode laser for both recording of HOEs and stable reference beam path modulation in the ESPI system. A temperature controlled diode laser (ONDAX) was used. Its nominal wavelength was $658 \mathrm{~nm}$ and the wavelength change and its associated power change per $\mathrm{mA}$ drive current were $5.1 \mathrm{pm}$ and $0.8 \mathrm{~mW}$ respectively.

The setup for recording the HOEs is shown in Fig. 1. A polarizing beam splitter (PBS) and two half wave plates (HWP1 \& 2) were used to split the laser beam into two parts with the same polarization state but variable beam ratio. The RHOE was recorded by the Denisyuk method (using beam 1 with the intensity of beam 2 set at zero), with a diffuser between the mirror and the emulsion to provide a speckled object beam. To record a THOE mirror 1 and the diffuser were removed, then the intensities of beams 1 and 2 were equalized. The two HOEs were recorded in this way to enable them to be used in contact with one another. Thus on reconstruction by beam 1 , a speckled reference beam is provided for the ESPI system by the RHOE and a beam diffracted by the THOE in the direction of beam 2 illuminates the test object. For RHOE recording PFG-03C silver halide emulsion on glass substrate $(6.3 \mathrm{~cm} \times 6.3 \mathrm{~cm})$, the laser beam intensity and exposure time was $500 \mu \mathrm{W} / \mathrm{cm}^{2}$ and $6 \mathrm{~s}$ respectively using the diode laser 
(Fig.1). SM- ${ }^{12}$ processing was used. The diffraction efficiency of the RHOE was $12 \%$. The THOE was recorded in HP-P ${ }^{13}$ silver halide holographic emulsion on glass substrate $(6.3 \mathrm{~cm} \times 6.3 \mathrm{~cm})$. The angle between the recording beams was $30^{\circ}$. The intensity and exposure time was $600 \mu \mathrm{W} / \mathrm{cm}^{2}$ and $5 \mathrm{~s}$ respectively; SM- $6^{12}$ processing was used. The diffraction efficiency of the THOE was $20 \%$.

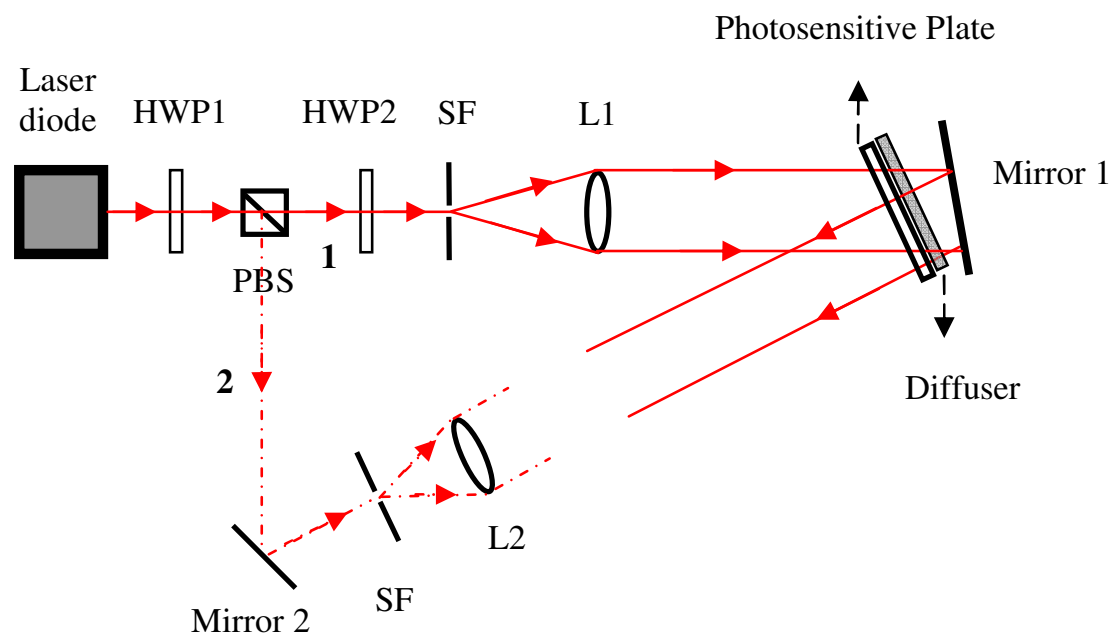

Fig. 1 Recording set up for HOEs: reflection hologram of a diffuser and transmission grating The ESPI system is shown in Fig. 2. The laser beam was spatially filtered (SF) and collimated (L2) to illuminate the RHOE at an angle of approximately $25^{\circ}$ to generate the speckled reference beam for the interferometer. The beam transmitted through the RHOE illuminates the THOE which diffracts the light to illuminate the test object. An unwanted secondary reflection grating is usually recoreded along with the THOE. To ensure that light diffracted by this grating does not enter the camera, the angle of illumination was altered slightly from the recording angle.. The object and reference beams were allowed to interfere on the focal plane of the CMOS camera (AVT Guppy F-036B) . The optical path difference between the object and reference beam is $2 l(26 \mathrm{~cm})$. A National Instruments digital to analog converter (D/A) (NI USB-6229) 
was used to provide two sinusoidal waveforms of same frequency but with different amplitude and phase, one to drive a PZT attached to the test object and the other to modulate the diode laser drive current. A rectangular pulse $(25 \mathrm{~Hz})$ triggers image acquisition by the camera. A rectangular waveform generated synchronously with the image acquisition pulse, causes the laser drive current to change, producing a phase change of $\pi$ in the interferometer at the beginning of each frame. Subtraction of the successive $\pi$ phase shifted speckle interferograms removes the background speckle noise ${ }^{3}$ and the resulting high contrast Bessel fringes are displayed on a monitor.

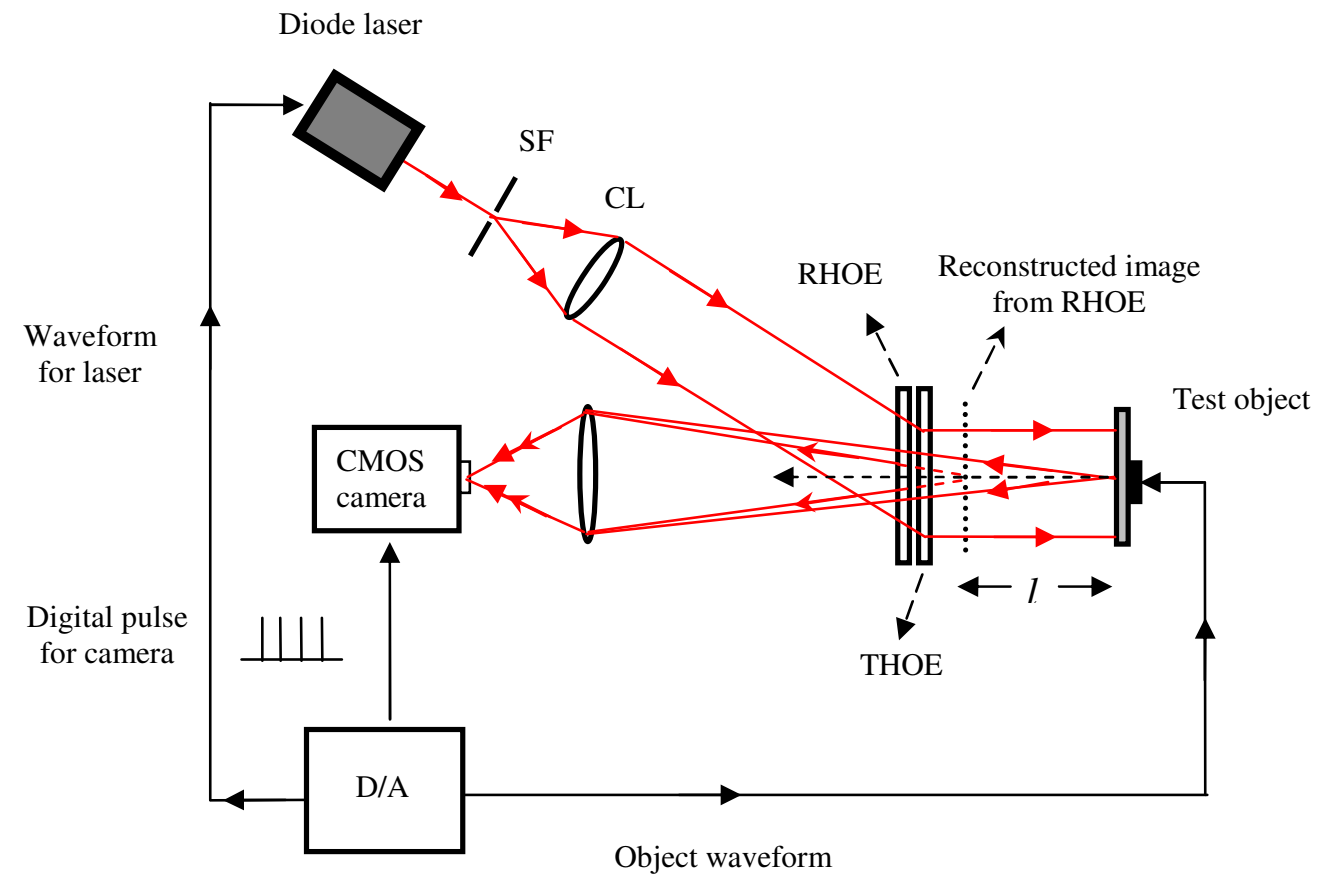

Fig. 2 Out-of-plane sensitive HOE based ESPI system

Simultaneously the optical path difference in the interferometer is modulated at the vibration frequency and at an amplitude which effectively freezes the motion of the object in certain regions for which the argument of the Bessel function in Eq. (3) is zero, and which therefore appear brightest in the display. 
The phase difference between the object and reference beams in an unbalanced interferometer with a path length difference $2 l$ is

$$
\Phi=\frac{2 \pi}{\lambda}(2 l)
$$

When the laser is modulated the relative phase difference changes by

$$
\Delta \Phi=-\frac{4 \pi l}{\lambda^{2}}\left(\frac{d \lambda}{d i}\right) \Delta i
$$

From Eq. 5 the amplitude of vibration on iso-amplitude contours ${ }^{3}$ is, ,

$$
a_{r}=\frac{2 l}{\lambda}\left(\frac{d \lambda}{d i}\right) \Delta i
$$

A circular metal plate, $5.4 \mathrm{~cm}$ in diameter was driven sinusoidally out of plane by a piezoelectric actuator at a resonant vibration frequency $5600 \mathrm{~Hz}$. The ordinary time average fringe pattern obtained by continuous $\pi$ shifted frame subtraction is shown in Fig. 3 (a). The brightest fringe was moved to a new position as seen in Fig. 3 (b) by modulating the laser wavelength with $\Delta \lambda=1.16 \mathrm{pm}$. From Eq. (6) amplitude of vibration in the brightest fringe regions in Fig. 3 (b) and (c) is $0.46 \mu \mathrm{m}$. Fig. 3 (c) shows the result when the phase of the reference beam modulation is altered by $180^{\circ}$
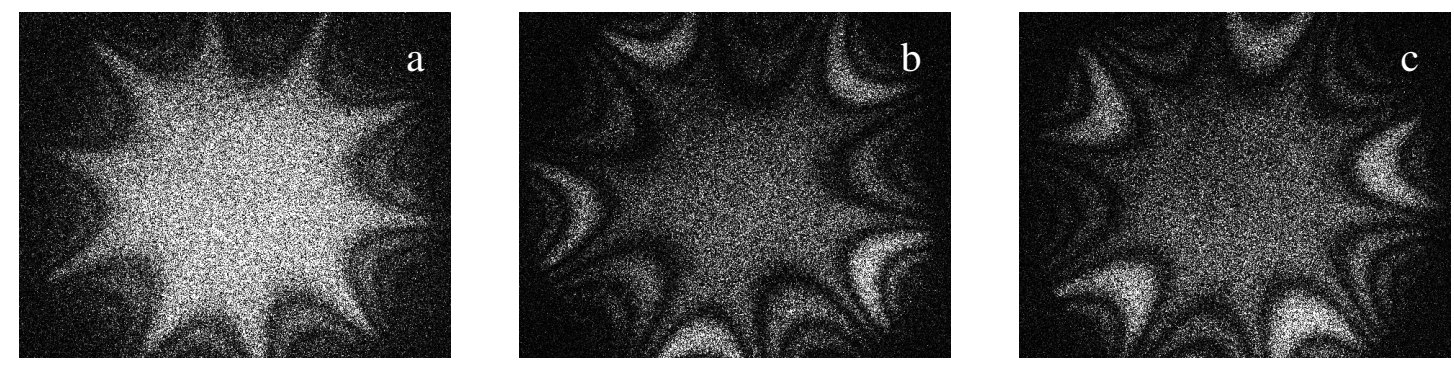

Fig. 3 Circular plate vibrating at $5600 \mathrm{~Hz}$ : (a) Ordinary time average fringes. Time average fringes by modulating the laser wavelength (b) $\Delta \lambda=1.16 \mathrm{pm}$ (c) $\Delta \lambda=1.16 \mathrm{pm}$ with $180^{\circ}$ phase shift. 
In conclusion a simple ESPI system using holographic optical elements to obtain vibrational amplitude and phase measurements was implemented. Use of HOEs reduces the number of optical elements drastically, leading to a compact ESPI system providing correlation fringes of good contrast.These ESPI systems can facilitate optical non-destructive testing at low cost for industrial use.

The authors would like to acknowledge Irish Technological Research Sector STRAND I and Enterprise Ireland Commercialisation Fund for financial support. We thank the School of Physics at the Dublin Institute of Technology. for laboratory facilities

\section{References}

1. C. Joenathan, Appl. Opt. 30, 4658 (1991)

2. F. M. Santoyo, M. C. Shellabear and J. R. Tyrer, Appl. Opt. 30, 717 (1991)

3. H. Atcha and R. P. Tatam, Meas. Sci. Technol. 5, 704 (1994)

4. A. Olszak, K. Patorski, Opt. Comms., 138, 265 (1997)

5. V. Petrov and B. Lau, Opt. Eng. 35, 2363 (1996)

6. M.P. Whelan, C. Forno, Proceedings of Interferometry '99, SPIE Vol. 3144, September (1999).

7. Toal V., Whelan W., Connelly M., Martin S., Reddy G. T. S. patent WO2009/044387 A2 (2009)

8. V Bavigadda, V Toal, R. Jallapuram and E. Mihaylova, Proc. SPIE, 7389 (2009)

9. S. R. Guntaka, V. Sainov, V.Toal, S. Martin, T. Petrova and J. Harizanova, J. Opt. A: Pure Appl. Opt. 8, 182 (2006) 
10. O. J. Lokberg and K. Hogmoen, J. Phys. E: Sci. Instr. 9, 847 (1976)

11. O. J. Lokberg and K.Hogmoen, Appl. Opt. 15, 2701 (1976)

12. S. R Guntaka, V. Toal and S. Martin, Appl. Opt. Appl. Opt. 41, 7475 (2002)

13. P. K. Rastogi, Editor, Digital speckle pattern interferometry and related techniques, pp. 231, John Wiley and Sons Ltd. (2001)

14. http://www.geola.1t/lt/holography_materials/chemistry/

15. http://www.optics.bas.bg/index.php?lang=en\&page=11 